\title{
Kinetic behaviors between acetone and composite bead in biofilter
}

\author{
Wu-Chung Chan • Liang-Yuan Chang
}

Published online: 21 February 2007

(C) Springer-Verlag 2007

Note from the Editors and Publisher on: Appl Microbiol Biotechnol

DOI: 10.1007/s00253-005-0236-y

Since the publication of this article, it has been brought to our attention that the same authors published a practically identical version of this paper in "Journal of Polymers and the Environment" (doi: 10.1007/S10924-006-0046-z) in 2006. The Editors and Springer would like to stress that this infraction runs contrary to our publishing ethics. Therefore, we distance ourselves from this article.

The online version of the original article can be found at http://dx.doi. org/10.1007/s00253-005-0236-y.

W.-C. Chan $(\bowtie) \cdot$ L.-Y. Chang

Civil Engineering Department, Chung-Hua University,

Hsinchu, Taiwan 3067, People's Republic of China

e-mail: wcchan@chu.edu.tw 